Provided for non-commercial research and education use. Not for reproduction, distribution or commercial use.

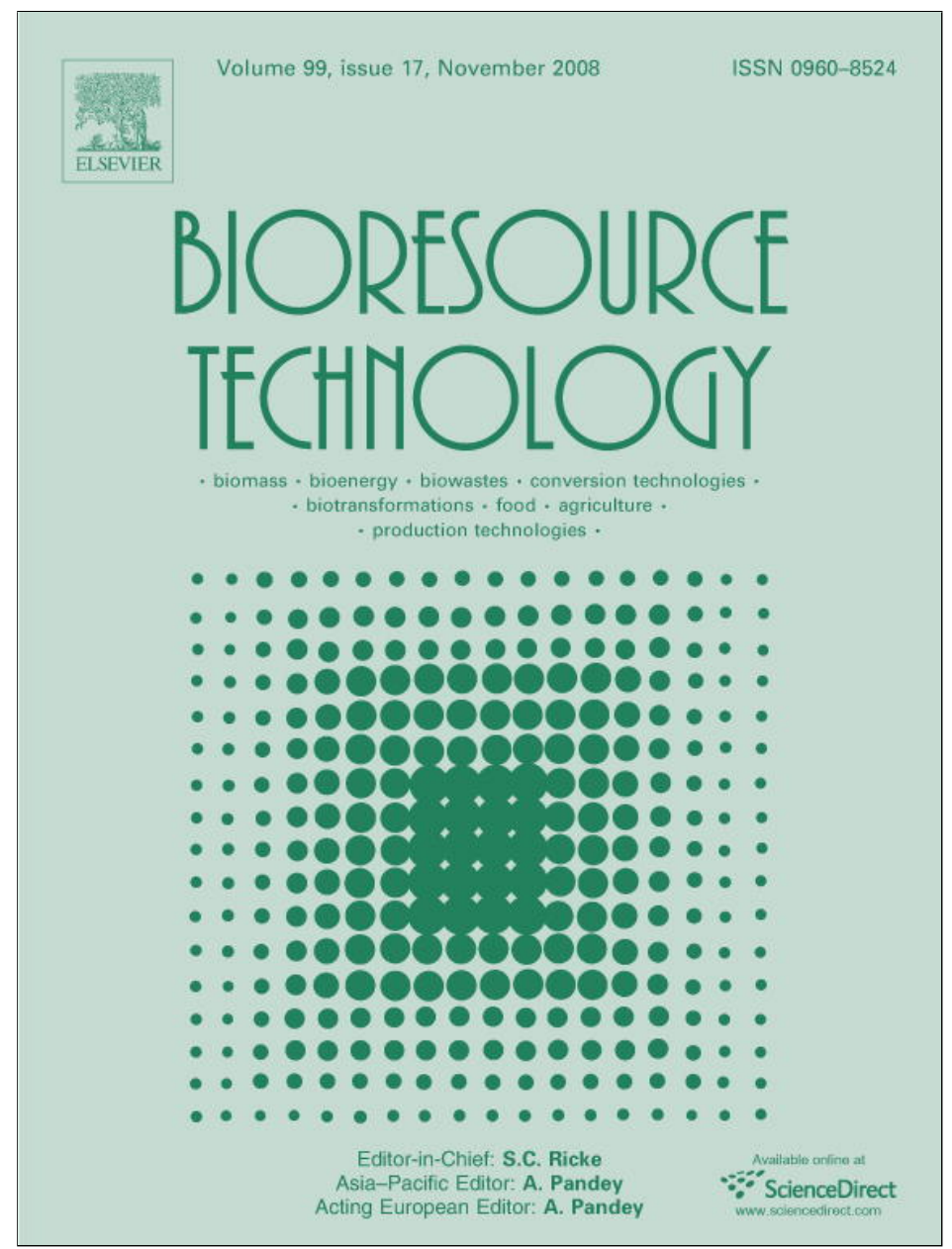

This article appeared in a journal published by Elsevier. The attached copy is furnished to the author for internal non-commercial research and education use, including for instruction at the authors institution and sharing with colleagues.

Other uses, including reproduction and distribution, or selling or licensing copies, or posting to personal, institutional or third party websites are prohibited.

In most cases authors are permitted to post their version of the article (e.g. in Word or Tex form) to their personal website or institutional repository. Authors requiring further information regarding Elsevier's archiving and manuscript policies are encouraged to visit:

http://www.elsevier.com/copyright 


\title{
Predicting anaerobic biogasification potential of ingestates and digestates of a full-scale biogas plant using chemical and biological parameters
}

\author{
Andrea Schievano, Michele Pognani, Giuliana D'Imporzano, Fabrizio Adani * \\ Dipartimento di Produzione Vegetale, Università degli Studi di Milano, Via Celoria 2, 20133 Milano, Italy
}

\section{A R T I C L E I N F O}

\section{Article history:}

Received 21 September 2007

Received in revised form 13 March 2008

Accepted 16 March 2008

Available online 28 April 2008

\section{Keywords:}

Biogas

Anaerobic digestion

Degradation yields

Anaerobic biogasification potential

Respirometric analysis

\begin{abstract}
A B S T R A C T
The aim of this work was to develop simple and fast tests to predict anaerobic biogasification potential (ABP) of ingestates and digestates from a biogas plant. Forty-six samples of both ingestates and digestates were collected within an eight-month observation period and were analyzed in terms of biological and chemical parameters, namely, ABP test, oxygen demand in a 20-h respirometric test (OD20), total solids (TS), volatile solids (VS), total organic carbon (TOC), total Kjeldahl nitrogen (TKN), ammonia, cell solubles (CS), acid detergent fibers (ADF), lignin (ADL), cellulose, and hemicellulose. Considering both quantitative (VS and TOC) and qualitative aspects (OD20 and CS) of organic matter (OM), four models (linear regressions; $0.80<R^{2}<0.913 ; 16 \%<$ standard errors $\left.<23 \%\right)$ were proposed to predict ABP. The models were chosen according to the needed accuracy of the evaluation in terms of time schedule and the availability of the required laboratory analyses.
\end{abstract}

(c) 2008 Elsevier Ltd. All rights reserved.

\section{Introduction}

Anaerobic digestion (AD) for biogas production could substitute for fossil fuel-derived energy and reduce environmental impacts by providing a clean fuel from renewable feedstock, such as energy crops, organic fractions of municipal solid wastes, and agro-industrial wastes (Chynoweth et al., 2001). Today, in northern Italy, because many biogas plants need to very quickly vary their feeding, depending on the availability of feedstock on the market, it would become necessary to predict the quality of a new ingestate mixture in just a few days. At the same time, ready responses on digestate slurries would give an evaluation of degradation yields achieved by the $\mathrm{AD}$ process.

Chemical characterization of both ingestates and digestates is still the most widely used method to evaluate process trends (Holm-Nielsen et al., 2006). While only a few parameters are determined by online monitoring (temperature, $\mathrm{pH}$ and biogas composition), fermentation process control is mostly achieved through manual sample extraction and time-consuming laboratory analyses (Sanderson et al., 1996; Wilman et al., 2000), such as total solids (TS), volatile solids (VS), total Kjeldahl nitrogen (TKN), ammonia, total organic carbon (TOC; Holm-Nielsen et al., 2006), total phosphorus $\left(\mathrm{P}_{2} \mathrm{O}_{5}\right)$, acid detergent fibers (ADF), acid detergent lignin (ADL), cellulose, hemicellulose, and cell solubles (CS; Gunaseelan, 1997; Zhang et al., 2007).

\footnotetext{
* Corresponding author. Tel.: +39 02503 16545; fax: +39 0250316521 .

E-mail address: fabrizio.adani@unimi.it (F. Adani).
}

Anaerobic biogasification potential (ABP) assay, also known as biochemical methane potential (BMP), can be used in evaluating biogas potential of both organic matrices composing an ingestate mixture and residual biogas in digestates. Moreover, such information allows a direct assessment of biogas production yields achieved by the AD process. On the other hand, such an assay lasts for at least $60 \mathrm{~d}$ (Adani et al., 2001; Hansen et al., 2004), requiring considerable laboratory work.

In the literature, some efforts have been done in studying the relationships between $\mathrm{ABP}$, digestibility, and chemical parameters of both fresh matrices and digested bioslurries (Bjorndal and Moore, 1985; Chandler et al., 1979; Gunaseelan, 2007; Han et al., 1975; Habig, 1985; Tong et al., 1990). Some interesting regression models predicting ABP through chemical composition can be found in Gunaseelan's recent work (Gunaseelan, 2007). Focusing only on some fresh lignocellulosic feedstock such as sorghum, napier grass and fruit, and vegetable solid wastes, some regressions were found between $\mathrm{ABP}$ and single chemical parameters.

Near-infrared spectroscopy (NIR) has been frequently reported as an easy and low-cost analysis, which could substitute for many laboratory analyses. Some authors focused on its possible use as an online technology for monitoring bioslurry compositions in biogas plants (Holm-Nielsen et al., 2006). Others reported regression models between NIR responses and chemical composition of biomass feedstock, mostly focusing on silages and crops for animal feed (Nousiainen et al., 2004; Sanderson et al., 1996; Wilman et al., 2000). On the other hand, to our knowledge, NIR has never been correlated to ABP. 
Biological analyses can also be used to predict ABP. Muller et al. (1998) indicated respirometric activity of municipal solid waste to be significantly correlated with anaerobic processes, i.e., biogas production from the same matrices.

The aim of this study was to determine regression models between $\mathrm{ABP}$ and some chemical and biological parameters of both ingestate mixtures and digested slurries of the observed biogas plant. This could enable a quick prediction of $A B P$ and readily give information for future monitoring and operating of the AD process of the biogas plant.

\section{Methods}

\subsection{Feedstock sample collection}

In 2006, Di.Pro.Ve. - University of Milan - Italy was involved in monitoring a full-scale biogas plant in northern Italy, consulting its operators regarding process start-up and optimization. A series of 46 samples was collected during an eight-mo plant-monitoring period (February-September 2006), with the aim of applying any positive result on operational management. The plant produced $2 \mathrm{MW}$ of electrical power by codigesting, in four continuously stir- red tank thermophilic reactors (CSTR) plus a post digester, a mixture of energetic crops, pig manure slurry, agro-industrial waste, and organic fraction of municipal solid waste (OFMSW).

Samples were divided into two groups. The first 12 samples were feed-in mixtures (ingestates) of the biogas plant. Due to practical requirements of the full-scale process, the composition of these mixtures significantly varied during the observation period. The second group included 34 samples representing the outputs of the five digesters after approximately 40 or $50 \mathrm{~d}$ of AD.

All the samples were dried for $24 \mathrm{~h}$ at $105^{\circ} \mathrm{C}$ (APHA, 1992) and were shredded in a blender to pass through a 2-mm mesh. Both biological and chemical analyses in this study were performed using the same dried and shredded samples.

\subsection{Analytical methods}

Representative samples were used to carry out all the analytical tests. TS, VS, and TOC were determined according to standard procedures (APHA, 1998). Ammonia and TKN (detected on fresh material) were determined according to the analytical method for wastewater sludges (IRSA CNR, 1994). Total phosphorus $\left(\mathrm{P}_{2} \mathrm{O}_{5}\right)$ content was determined using the standard methods for the exam-

Table 1

Chemical characterization of the analyzed samples

\begin{tabular}{|c|c|c|c|c|c|c|c|c|c|}
\hline Date & $\mathrm{RT}^{\mathrm{a}}$ (Days) & TS (\%FM) & VS (\%TS) & TOC (\%TS) & Ammonia (\%TS) & TKN (\%TS) & $\mathrm{P}_{2} \mathrm{O}_{5}$ (\%TS) & TOC/TKN & Ammonia/TKN \\
\hline $1 / 2 / 2006$ & 0 & $13.80 \pm 0.20$ & $92.40 \pm 0.20$ & $40.80 \pm 0.10$ & 0.47 & $2.75 \pm 0.04$ & $0.45 \pm 0.01$ & 14.84 & 0.17 \\
\hline $9 / 2 / 2006$ & 0 & $12.70 \pm 0.50$ & $91.80 \pm 0.50$ & $44.50 \pm 0.30$ & 0.75 & $3.32 \pm 0.05$ & $0.65 \pm 0.09$ & 13.40 & 0.23 \\
\hline $15 / 02 / 06$ & 0 & $11.92 \pm 0.40$ & $90.90 \pm 0.30$ & $47.20 \pm 0.20$ & 0.81 & $3.51 \pm 0.05$ & $0.59 \pm 0.00$ & 13.45 & 0.23 \\
\hline $23 / 02 / 06$ & 0 & $11.98 \pm 0.70$ & $92.10 \pm 0.60$ & $43.00 \pm 1.00$ & 0.63 & $3.09 \pm 0.10$ & $0.65 \pm 0.01$ & 13.92 & 0.20 \\
\hline $2 / 3 / 2006$ & 0 & $14.70 \pm 1.00$ & $91.80 \pm 1.10$ & $44.50 \pm 0.70$ & 0.49 & $2.98 \pm 0.03$ & $0.83 \pm 0.11$ & 14.93 & 0.16 \\
\hline $8 / 3 / 2006$ & 0 & $12.24 \pm 0.80$ & $92.00 \pm 0.30$ & $42.30 \pm 0.70$ & 0.64 & $3.05 \pm 0.10$ & $0.81 \pm 0.13$ & 13.87 & 0.21 \\
\hline $22 / 03 / 06$ & 0 & $13.51 \pm 1.10$ & $92.40 \pm 0.80$ & $44.10 \pm 0.50$ & 0.67 & $3.53 \pm 0.04$ & $0.86 \pm 0.03$ & 12.49 & 0.19 \\
\hline $27 / 03 / 06$ & 0 & $11.08 \pm 0.60$ & $89.00 \pm 1.50$ & $43.00 \pm 0.40$ & 0.64 & $3.73 \pm 0.00$ & $0.95 \pm 0.08$ & 11.53 & 0.17 \\
\hline $3 / 5 / 2006$ & 0 & $12.29 \pm 0.20$ & $93.00 \pm 1.00$ & $45.60 \pm 0.00$ & 1.00 & $3.67 \pm 0.05$ & $0.54 \pm 0.11$ & 12.43 & 0.27 \\
\hline $31 / 05 / 06$ & 0 & $15.03 \pm 0.50$ & $81.00 \pm 0.80$ & $43.80 \pm 0.30$ & 1.39 & $5.15 \pm 0.08$ & $1.77 \pm 0.07$ & 8.50 & 0.27 \\
\hline $1 / 8 / 2006$ & 0 & $19.56 \pm 1.10$ & $93.10 \pm 0.10$ & $51.50 \pm 0.70$ & 1.05 & $1.96 \pm 0.01$ & $0.48 \pm 0.01$ & 14.88 & 0.30 \\
\hline $22 / 08 / 06$ & 0 & $14.03 \pm 0.30$ & $89.60 \pm 0.50$ & $42.70 \pm 0.20$ & 0.06 & $3.18 \pm 0.01$ & $0.62 \pm 0.07$ & 13.43 & 0.33 \\
\hline $1 / 2 / 2006$ & 40 & $9.58 \pm 0.80$ & $77.40 \pm 0.60$ & $35.80 \pm 0.90$ & 1.80 & $4.94 \pm 0.05$ & $1.90 \pm 0.31$ & 7.25 & 0.36 \\
\hline $1 / 2 / 2006$ & 40 & $8.92 \pm 1.50$ & $72.20 \pm 0.80$ & $30.10 \pm 1.00$ & 2.37 & $5.58 \pm 0.04$ & $3.37 \pm 0.25$ & 5.39 & 0.42 \\
\hline $9 / 2 / 2006$ & 40 & $9.08 \pm 1.00$ & $72.30 \pm 1.00$ & $37.90 \pm 1.20$ & 1.74 & $5.26 \pm 0.02$ & $1.67 \pm 0.20$ & 7.21 & 0.33 \\
\hline $9 / 2 / 2006$ & 40 & $5.46 \pm 0.80$ & $67.20 \pm 0.20$ & $33.60 \pm 1.70$ & 3.32 & $6.82 \pm 0.09$ & $1.87 \pm 0.21$ & 4.93 & 0.49 \\
\hline $15 / 02 / 06$ & 40 & $3.62 \pm 0.10$ & $73.30 \pm 0.10$ & $31.90 \pm 0.80$ & 2.40 & $5.97 \pm 0.13$ & $1.50 \pm 0.09$ & 5.34 & 0.40 \\
\hline $23 / 02 / 06$ & 40 & $8.26 \pm 0.50$ & $79.30 \pm 0.50$ & $43.00 \pm 0.80$ & 1.88 & $4.43 \pm 0.15$ & $2.61 \pm 0.36$ & 9.71 & 0.42 \\
\hline $23 / 02 / 06$ & 40 & $8.21 \pm 0.60$ & $68.60 \pm 1.10$ & $37.80 \pm 0.00$ & 2.23 & $5.26 \pm 0.03$ & $3.29 \pm 0.05$ & 7.19 & 0.42 \\
\hline $2 / 3 / 2006$ & 40 & $8.47 \pm 0.80$ & $75.70 \pm 0.20$ & $39.20 \pm 0.20$ & 1.78 & $5.26 \pm 0.37$ & $2.14 \pm 0.20$ & 7.45 & 0.34 \\
\hline $2 / 3 / 2006$ & 40 & $8.10 \pm 1.00$ & $77.40 \pm 0.50$ & $33.80 \pm 0.10$ & 1.96 & $4.56 \pm 0.02$ & $2.75 \pm 0.38$ & 7.41 & 0.43 \\
\hline $8 / 3 / 2006$ & 40 & $8.39 \pm 0.20$ & $81.90 \pm 0.80$ & $39.50 \pm 0.90$ & 2.16 & $5.39 \pm 0.19$ & $2.45 \pm 0.02$ & 7.33 & 0.40 \\
\hline $8 / 3 / 2006$ & 40 & $7.09 \pm 0.10$ & $78.60 \pm 1.50$ & $36.90 \pm 0.70$ & 2.92 & $6.37 \pm 0.01$ & $2.28 \pm 0.05$ & 5.79 & 0.46 \\
\hline $27 / 03 / 06$ & 40 & $5.16 \pm 0.50$ & $75.40 \pm 0.60$ & $37.50 \pm 1.10$ & 4.26 & $7.39 \pm 0.13$ & $2.38 \pm 0.12$ & 5.07 & 0.58 \\
\hline $27 / 03 / 06$ & 40 & $5.28 \pm 1.10$ & $75.70 \pm 2.00$ & $40.20 \pm 2.00$ & 4.34 & $7.66 \pm 0.02$ & $2.09 \pm 0.00$ & 5.25 & 0.57 \\
\hline $3 / 5 / 2006$ & 40 & $6.45 \pm 0.20$ & $75.70 \pm 1.50$ & $38.50 \pm 0.40$ & 3.90 & $6.58 \pm 0.03$ & $2.02 \pm 0.12$ & 5.85 & 0.59 \\
\hline $31 / 05 / 06$ & 40 & $8.57 \pm 0.50$ & $71.70 \pm 0.60$ & $37.00 \pm 0.70$ & 3.25 & $6.61 \pm 0.15$ & $2.88 \pm 0.26$ & 5.60 & 0.49 \\
\hline $31 / 05 / 06$ & 40 & $9.16 \pm 0.80$ & $70.40 \pm 0.20$ & $35.30 \pm 0.40$ & 3.07 & $7.05 \pm 0.12$ & $2.58 \pm 0.04$ & 5.01 & 0.44 \\
\hline $31 / 05 / 06$ & 40 & $12.92 \pm 1.50$ & $76.10 \pm 0.40$ & $40.90 \pm 0.80$ & 2.05 & $4.96 \pm 0.10$ & $2.84 \pm 0.28$ & 8.25 & 0.41 \\
\hline $11 / 7 / 2006$ & 40 & $2.00 \pm 0.60$ & $70.50 \pm 1.10$ & $36.40 \pm 0.20$ & 4.02 & $8.48 \pm 0.07$ & $2.46 \pm 0.04$ & 4.29 & 0.47 \\
\hline $22 / 08 / 06$ & 40 & $6.39 \pm 2.00$ & $74.80 \pm 0.60$ & $41.60 \pm 0.20$ & 2.67 & $5.42 \pm 0.09$ & $2.18 \pm 0.00$ & 7.68 & 0.49 \\
\hline $22 / 08 / 06$ & 40 & $4.09 \pm 1.50$ & $73.60 \pm 0.20$ & $36.60 \pm 0.20$ & 6.15 & $9.12 \pm 0.05$ & $2.41 \pm 0.16$ & 4.01 & 0.67 \\
\hline $22 / 08 / 06$ & 40 & $7.26 \pm 0.60$ & $77.00 \pm 0.70$ & $42.90 \pm 1.80$ & 2.33 & $5.03 \pm 0.12$ & $1.79 \pm 0.09$ & 8.53 & 0.46 \\
\hline $6 / 9 / 2006$ & 40 & $3.28 \pm 0.20$ & $90.90 \pm 0.20$ & $33.40 \pm 0.50$ & 6.79 & $10.82 \pm 0.07$ & $2.25 \pm 0.01$ & 3.09 & 0.63 \\
\hline $6 / 9 / 2006$ & 40 & $5.04 \pm 0.40$ & $71.00 \pm 1.00$ & $37.80 \pm 0.00$ & 5.79 & $8.71 \pm 0.02$ & $2.00 \pm 0.11$ & 4.34 & 0.66 \\
\hline $6 / 9 / 2006$ & 40 & $4.62 \pm 1.10$ & $68.70 \pm 1.10$ & $36.70 \pm 1.80$ & 6.43 & $9.52 \pm 0.17$ & $2.50 \pm 0.00$ & 3.86 & 0.68 \\
\hline $8 / 9 / 2006$ & 40 & $7.01 \pm 0.60$ & $79.40 \pm 0.20$ & $38.80 \pm 0.40$ & 3.87 & $6.05 \pm 0.02$ & $2.66 \pm 0.18$ & 6.41 & 0.64 \\
\hline $8 / 9 / 2006$ & 40 & $5.40 \pm 0.20$ & $76.00 \pm 0.50$ & $39.40 \pm 2.10$ & 4.80 & $7.52 \pm 0.03$ & $2.11 \pm 0.03$ & 5.24 & 0.64 \\
\hline $13 / 09 / 06$ & 40 & $3.72 \pm 0.70$ & $69.90 \pm 0.20$ & $37.10 \pm 0.80$ & 6.71 & $10.52 \pm 0.06$ & $2.21 \pm 0.14$ & 3.53 & 0.64 \\
\hline $13 / 09 / 06$ & 40 & $5.26 \pm 0.20$ & $73.30 \pm 0.50$ & $37.80 \pm 1.00$ & 4.82 & $7.71 \pm 0.01$ & $2.78 \pm 0.13$ & 4.90 & 0.63 \\
\hline $15 / 09 / 06$ & 40 & $4.61 \pm 1.00$ & $72.90 \pm 0.40$ & $39.40 \pm 0.00$ & 5.47 & $8.56 \pm 0.02$ & $2.11 \pm 0.07$ & 4.60 & 0.64 \\
\hline 15/09/06 & 40 & $5.27 \pm 1.10$ & $71.70 \pm 0.70$ & $37.60 \pm 0.10$ & 4.88 & $7.67 \pm 0.02$ & $2.62 \pm 0.10$ & 4.90 & 0.64 \\
\hline $11 / 7 / 2006$ & 50 & $3.50 \pm 0.20$ & $70.20 \pm 1.00$ & $39.00 \pm 1.20$ & 6.60 & $10.20 \pm 0.01$ & $2.87 \pm 0.04$ & 3.82 & 0.65 \\
\hline $22 / 08 / 06$ & 50 & $3.00 \pm 0.50$ & $68.40 \pm 0.80$ & $37.75 \pm 0.20$ & 8.19 & $11.00 \pm 0.03$ & $3.01 \pm 0.21$ & 3.43 & 0.74 \\
\hline $13 / 09 / 06$ & 50 & $4.40 \pm 0.20$ & $71.30 \pm 1.10$ & $36.40 \pm 0.20$ & 5.66 & $8.90 \pm 0.01$ & $2.23 \pm 0.01$ & 4.09 & 0.64 \\
\hline $15 / 09 / 06$ & 50 & $3.48 \pm 0.50$ & $71.20 \pm 0.60$ & $36.10 \pm 0.10$ & 6.63 & $10.60 \pm 0.04$ & $2.32 \pm 0.03$ & 3.41 & 0.63 \\
\hline
\end{tabular}

a Retention time into the anaerobic digesters. 
ination of water and wastewater (APHA, 1998). Fiber analyses were performed for neutral detergent fiber (NDF), neutral detergent acid detergent fibers (NDADF), and acid detergent lignin (ADL), following Van Soest's method (Van Soest et al., 1991). Cell solubles (CS), lignin plus unhydrolyzable lipid (ADL), cellulose (NDADF-ADL), and hemicellulose (NDF-NDADF) were calculated according to Van Soest et al. (1991). All analyses were performed in duplicates.

\subsection{Anaerobic biogasification potential (ABP) assay}

\subsubsection{Seed inoculum preparation}

Inoculum in stable methanogenic activity $\left(\mathrm{CH}_{4}>60 \%\right.$ in biogas, $\mathrm{v} / \mathrm{v}$ ) was obtained using the output digestate of the post digester of the plant. The $\mathrm{pH}$ was around 7.8; TS and VS contents were about $3-4 \%$ on wet weight basis $(\mathrm{w} / \mathrm{w})$ and $70-80 \%$ on TS basis, respectively. Digestate was incubated at $37 \pm 1^{\circ} \mathrm{C}$ for $15 \mathrm{~d}$ before use.

\subsubsection{ABP assay}

The ABP of all samples was determined using the method of Adani et al. (2001) with a few modifications developed, according to Hansen et al. (2004). In 100-ml serum bottles, $0.62 \mathrm{~g}$ of dried sample was added to $37.5 \mathrm{ml}$ of inoculum and $22 \mathrm{ml}$ of deionized water. The batch tests were carried out with $60-\mathrm{ml}$ samples (about $3.5 \% \mathrm{TS}$ ) and $40 \mathrm{ml}$ of headspace. The fresh feedstock and inoculum percentages of TS were $35 \%$ and $65 \%$, respectively. Control blanks were prepared using $60 \mathrm{ml}$ of inoculum.

All batches were sealed with teflon hermetic caps, flushed with a $\mathrm{N}_{2}$ atmosphere, and incubated at $37 \pm 1^{\circ} \mathrm{C}$, until no further biogas production was detected (normally around $60 \mathrm{~d}$ ). Assay bottles were periodically analyzed for both quantitative and qualitative determination of biogas production. Quantitative biogas production was estimated by withdrawing extra-pressure gas with a $60-\mathrm{ml}$ syringe. Biogas production of blank control batches was subtracted from biogas production of every sample. Qualitative characterization of biogas was performed by a gas chromatograph (Carlo Erba Megaseries 5300, capillary column 25-m $\times 0.32-\mathrm{mm}$ diameter and flame ionization detector (FID)) to determine $\mathrm{CH}_{4}$ $\mathrm{CO}_{2}$ ratio in the biogas. The carrier gas was nitrogen at $20 \mathrm{kPa}$ pressure and temperatures of injector and FID were 130 and $150{ }^{\circ} \mathrm{C}$, respectively. Comparison of obtained peak areas was carried out with a standard gas mixture of $30: 70 \mathrm{CH}_{4}: \mathrm{CO}_{2}$. All tests were run in duplicates.

Table 2

Fibers content and results of biological tests (ABP and OD20) on dry matter

\begin{tabular}{|c|c|c|c|c|c|c|c|c|}
\hline Date & $\mathrm{RT}^{\mathrm{a}}$ (Days) & ADF (\%TS) & ADL (\%TS) & Hemicellulose (\%TS) & Cellulose (\%TS) & CS (\%TS) & OD $20\left(\mathrm{mg} \mathrm{O}_{2} \mathrm{~g}^{-1} \mathrm{TS}\right)$ & $\mathrm{ABP}\left(\mathrm{Nml} \mathrm{g}^{-1} \mathrm{TS}\right)$ \\
\hline $1 / 2 / 2006$ & 0 & $7.6 \pm 0.3$ & $3.1 \pm 0.1$ & $2.8 \pm 0.1$ & $4.5 \pm 0.2$ & $89.7 \pm 3.1$ & $164 \pm 5.7$ & $608 \pm 6$ \\
\hline $9 / 2 / 2006$ & 0 & $12.9 \pm 0.8$ & $6.7 \pm 0.4$ & $3.2 \pm 0.2$ & $6.2 \pm 0.4$ & $83.9 \pm 5.3$ & $143 \pm 9.1$ & $636 \pm 2$ \\
\hline $15 / 02 / 06$ & 0 & $13.3 \pm 1.1$ & $8.0 \pm 0.7$ & $4.1 \pm 0.3$ & $5.3 \pm 0.4$ & $82.7 \pm 6.8$ & $160 \pm 13.2$ & $627 \pm 6$ \\
\hline $23 / 02 / 06$ & 0 & $8.9 \pm 0.5$ & $4.0 \pm 0.2$ & $2.9 \pm 0.2$ & $4.9 \pm 0.3$ & $88.2 \pm 4.8$ & $218 \pm 11.9$ & $498 \pm 26$ \\
\hline $2 / 3 / 2006$ & 0 & $9.9 \pm 0.1$ & $4.6 \pm 0.1$ & $3.8 \pm 0.1$ & $5.2 \pm 0.1$ & $86.4 \pm 1.3$ & $175 \pm 2.5$ & $692 \pm 11$ \\
\hline $8 / 3 / 2006$ & 0 & $7.1 \pm 0.2$ & $3.2 \pm 0.1$ & $3.9 \pm 0.1$ & $4.0 \pm 0.1$ & $89.0 \pm 3.1$ & $166 \pm 5.7$ & $720 \pm 23$ \\
\hline $22 / 03 / 06$ & 0 & $9.2 \pm 0.6$ & $4.6 \pm 0.3$ & $4.2 \pm 0.3$ & $4.6 \pm 0.3$ & $86.5 \pm 5.5$ & $275 \pm 17.4$ & $715 \pm 7$ \\
\hline $27 / 03 / 06$ & 0 & $8.8 \pm 0.7$ & $4.6 \pm 0.4$ & $3.4 \pm 0.3$ & $5.2 \pm 0.4$ & $86.8 \pm 7.2$ & $305 \pm 25.1$ & $700 \pm 19$ \\
\hline $3 / 5 / 2006$ & 0 & $25.3 \pm 1.4$ & $4.0 \pm 0.2$ & $5.4 \pm 0.3$ & $4.8 \pm 0.3$ & $85.8 \pm 4.7$ & $279 \pm 15.2$ & $772 \pm 34$ \\
\hline $31 / 05 / 06$ & 0 & $18.9 \pm 0.3$ & $15.0 \pm 0.2$ & $3.9 \pm 0.1$ & $10.4 \pm 0.2$ & $70.7 \pm 1.0$ & $310 \pm 4.5$ & $588 \pm 6$ \\
\hline $1 / 8 / 2006$ & 0 & $16.6 \pm 2.2$ & $23.7 \pm 3.2$ & $0.0 \pm 0.0$ & $9.0 \pm 1.2$ & $67.3 \pm 9.1$ & $200 \pm 26.9$ & $619 \pm 11$ \\
\hline $22 / 08 / 06$ & 0 & $17.8 \pm 0.6$ & $9.8 \pm 0.3$ & $2.9 \pm 0.1$ & $9.7 \pm 0.3$ & $77.6 \pm 2.7$ & $235 \pm 8.1$ & $582 \pm 14$ \\
\hline $1 / 2 / 2006$ & 40 & $43.9 \pm 2.8$ & $24.8 \pm 1.6$ & $11.7 \pm 0.7$ & $19.1 \pm 1.2$ & $44.4 \pm 2.8$ & $68.2 \pm 4.3$ & $194 \pm 4$ \\
\hline $1 / 2 / 2006$ & 40 & $34.9 \pm 2.9$ & $22.5 \pm 1.9$ & $9.9 \pm 0.8$ & $12.4 \pm 1.0$ & $55.2 \pm 4.6$ & $62.5 \pm 5.2$ & $115 \pm 1$ \\
\hline $9 / 2 / 2006$ & 40 & $20.5 \pm 1.1$ & $12.6 \pm 0.7$ & $10.5 \pm 0.6$ & $7.9 \pm 0.4$ & $69.1 \pm 3.8$ & $85.7 \pm 4.7$ & $213 \pm 1$ \\
\hline $9 / 2 / 2006$ & 40 & $13.4 \pm 0.2$ & $10.7 \pm 0.2$ & $13.0 \pm 0.2$ & $2.7 \pm 0.0$ & $73.6 \pm 1.1$ & $84.3 \pm 1.2$ & $153 \pm 6$ \\
\hline $15 / 02 / 06$ & 40 & $14.2 \pm 0.5$ & $12.4 \pm 0.4$ & $13.2 \pm 0.5$ & $1.8 \pm 0.1$ & $72.6 \pm 2.5$ & $95.7 \pm 3.3$ & $215 \pm 6$ \\
\hline $23 / 02 / 06$ & 40 & $21.9 \pm 1.4$ & $13.0 \pm 0.8$ & $9.8 \pm 0.6$ & $8.9 \pm 0.6$ & $68.3 \pm 4.3$ & $90.5 \pm 5.7$ & $380 \pm 10$ \\
\hline $23 / 02 / 06$ & 40 & $22.8 \pm 1.9$ & $14.4 \pm 1.2$ & $13.9 \pm 1.1$ & $8.4 \pm 0.7$ & $63.3 \pm 5.2$ & $122 \pm 10.1$ & $235 \pm 4$ \\
\hline $2 / 3 / 2006$ & 40 & $16.8 \pm 0.9$ & $11.5 \pm 0.6$ & $9.4 \pm 0.5$ & $5.3 \pm 0.3$ & $73.8 \pm 4.0$ & $81.2 \pm 4.4$ & $405 \pm 26$ \\
\hline $2 / 3 / 2006$ & 40 & $35.9 \pm 0.5$ & $17.3 \pm 0.3$ & $8.8 \pm 0.1$ & $18.6 \pm 0.3$ & $55.4 \pm 0.8$ & $64.3 \pm 0.9$ & $240 \pm 9$ \\
\hline $8 / 3 / 2006$ & 40 & $21.2 \pm 2.9$ & $12.5 \pm 1.7$ & $9.5 \pm 1.3$ & $8.7 \pm 1.2$ & $69.3 \pm 9.3$ & $143 \pm 19.3$ & $369 \pm 14$ \\
\hline $8 / 3 / 2006$ & 40 & $21.2 \pm 0.7$ & $14.2 \pm 0.5$ & $11.8 \pm 0.4$ & $7.0 \pm 0.2$ & $67.0 \pm 2.3$ & $91.9 \pm 3.2$ & $245 \pm 21$ \\
\hline $27 / 03 / 06$ & 40 & $32.5 \pm 2.1$ & $21.3 \pm 1.4$ & $7.2 \pm 0.5$ & $11.2 \pm 0.7$ & $60.4 \pm 3.8$ & $116 \pm 7.4$ & $333 \pm 13$ \\
\hline $27 / 03 / 06$ & 40 & $31.0 \pm 2.6$ & $21.3 \pm 1.8$ & $7.4 \pm 0.6$ & $9.7 \pm 0.8$ & $61.6 \pm 5.1$ & $113 \pm 9.3$ & $283 \pm 12$ \\
\hline $3 / 5 / 2006$ & 40 & $45.1 \pm 2.5$ & $32.9 \pm 1.8$ & $11.3 \pm 0.6$ & $12.2 \pm 0.7$ & $43.6 \pm 2.4$ & $98.8 \pm 5.4$ & $224 \pm 3$ \\
\hline $31 / 05 / 06$ & 40 & $23.1 \pm 0.3$ & $15.6 \pm 0.2$ & $8.5 \pm 0.1$ & $7.5 \pm 0.1$ & $68.3 \pm 1.0$ & $98.5 \pm 1.4$ & $319 \pm 23$ \\
\hline $31 / 05 / 06$ & 40 & $24.1 \pm 3.2$ & $17.6 \pm 2.4$ & $7.8 \pm 1.0$ & $6.5 \pm 0.9$ & $68.1 \pm 9.2$ & $135 \pm 18.2$ & $252 \pm 9$ \\
\hline 31/05/06 & 40 & $27.2 \pm 0.9$ & $19.2 \pm 0.7$ & $7.7 \pm 0.3$ & $8.0 \pm 0.3$ & $65.1 \pm 2.2$ & $128 \pm 4.4$ & $416 \pm 3$ \\
\hline $11 / 7 / 2006$ & 40 & $26.5 \pm 1.7$ & $20.8 \pm 1.3$ & $4.5 \pm 0.3$ & $5.7 \pm 0.4$ & $69.0 \pm 4.4$ & $64.8 \pm 4.1$ & $286 \pm 11$ \\
\hline $22 / 08 / 06$ & 40 & $27.7 \pm 2.3$ & $19.2 \pm 1.6$ & $2.6 \pm 0.2$ & $8.5 \pm 0.7$ & $69.8 \pm 5.8$ & $102 \pm 8.4$ & $412 \pm 5$ \\
\hline $22 / 08 / 06$ & 40 & $29.8 \pm 1.6$ & $21.8 \pm 1.2$ & $1.4 \pm 0.1$ & $8.0 \pm 0.4$ & $68.8 \pm 3.7$ & $71.2 \pm 3.9$ & $312 \pm 0$ \\
\hline $22 / 08 / 06$ & 40 & $41.3 \pm 0.6$ & $29.3 \pm 0.4$ & $0.0 \pm 0.0$ & $12.0 \pm 0.2$ & $60.2 \pm 0.9$ & $101 \pm 1.5$ & $437 \pm 37$ \\
\hline $6 / 9 / 2006$ & 40 & $29.1 \pm 6.8$ & $21.5 \pm 5.0$ & $0.8 \pm 0.2$ & $7.6 \pm 1.8$ & $70.1 \pm 16.4$ & $79.3 \pm 18.6$ & $265 \pm 6$ \\
\hline $6 / 9 / 2006$ & 40 & $41.0 \pm 1.4$ & $30.7 \pm 1.1$ & $0.0 \pm 0.0$ & $10.3 \pm 0.4$ & $60.0 \pm 2.1$ & $58.2 \pm 2.0$ & $232 \pm 10$ \\
\hline $6 / 9 / 2006$ & 40 & $36.7 \pm 2.3$ & $26.4 \pm 1.7$ & $1.5 \pm 0.1$ & $10.3 \pm 0.7$ & $61.8 \pm 3.9$ & $91.1 \pm 5.8$ & $188 \pm 6$ \\
\hline $8 / 9 / 2006$ & 40 & $39.8 \pm 3.3$ & $27.5 \pm 2.3$ & $0.0 \pm 0.0$ & $12.3 \pm 1.0$ & $58.1 \pm 4.8$ & $99 \pm 8.2$ & $242 \pm 3$ \\
\hline $8 / 9 / 2006$ & 40 & $34.1 \pm 1.9$ & $24.7 \pm 1.3$ & $4.6 \pm 0.3$ & $9.4 \pm 0.5$ & $61.4 \pm 3.3$ & $79.2 \pm 4.3$ & $279 \pm 3$ \\
\hline $13 / 09 / 06$ & 40 & $30.4 \pm 0.4$ & $22.2 \pm 0.3$ & $0.0 \pm 0.0$ & $8.2 \pm 0.1$ & $72.2 \pm 1.0$ & $129 \pm 1.9$ & $244 \pm 8$ \\
\hline $13 / 09 / 06$ & 40 & $33.0 \pm 4.4$ & $23.5 \pm 3.2$ & $4.4 \pm 0.6$ & $9.5 \pm 1.3$ & $62.6 \pm 8.4$ & $104 \pm 14.0$ & $244 \pm 6$ \\
\hline $15 / 09 / 06$ & 40 & $31.3 \pm 1.1$ & $21.5 \pm 0.7$ & $8.6 \pm 0.3$ & $9.8 \pm 0.3$ & $60.1 \pm 2.1$ & $88 \pm 3.0$ & $268 \pm 4$ \\
\hline $15 / 09 / 06$ & 40 & $30.9 \pm 2.0$ & $21.3 \pm 1.4$ & $0.5 \pm 0.0$ & $9.6 \pm 0.6$ & $68.7 \pm 4.4$ & $93 \pm 5.9$ & $274 \pm 13$ \\
\hline $11 / 7 / 2006$ & 50 & $36.5 \pm 3.0$ & $27.1 \pm 2.2$ & $5.3 \pm 0.4$ & $9.4 \pm 0.8$ & $58.0 \pm 4.8$ & $13.6 \pm 1.1$ & $66 \pm 1$ \\
\hline $22 / 08 / 06$ & 50 & $27.9 \pm 1.5$ & $22.1 \pm 1.2$ & $11.6 \pm 0.6$ & $5.8 \pm 0.3$ & $60.5 \pm 3.3$ & $29.9 \pm 1.6$ & $77 \pm 8$ \\
\hline $13 / 09 / 06$ & 50 & $34.0 \pm 0.5$ & $24.4 \pm 0.4$ & $5.0 \pm 0.1$ & $9.6 \pm 0.1$ & $61.0 \pm 0.9$ & $77.2 \pm 1.1$ & $235 \pm 6$ \\
\hline $15 / 09 / 06$ & 50 & $27.8 \pm 6.5$ & $19.7 \pm 4.6$ & $0.7 \pm 0.2$ & $8.1 \pm 1.9$ & $71.5 \pm 16.8$ & $128 \pm 30.0$ & $245 \pm 12$ \\
\hline
\end{tabular}

a Retention time into the anaerobic digesters. 
Table 3

ANOVA applied on the two groups of samples (ingestates and digestates)

\begin{tabular}{|c|c|c|c|c|}
\hline Parameter & Groups & Mean $^{a}$ & Minimum & Maximum \\
\hline \multirow[t]{2}{*}{ TS (\%FM) } & Ingestates & $13.6 \pm 2.2 \mathrm{~b}$ & 11.1 & 19.6 \\
\hline & Digestates & $6.2 \pm 2.4 \mathrm{a}$ & 2.0 & 12.9 \\
\hline \multirow[t]{2}{*}{ VS (\%TS) } & Ingestates & $90.8 \pm 3.3 \mathrm{~b}$ & 81.0 & 93.1 \\
\hline & Digestates & $73.8 \pm 3.8 \mathrm{a}$ & 67.2 & 81.9 \\
\hline \multirow[t]{2}{*}{ TOC (\%TS) } & Ingestates & $44.4 \pm 2.8 \mathrm{~b}$ & 40.8 & 51.5 \\
\hline & Digestates & $37.5 \pm 2.8 \mathrm{a}$ & 30.1 & 43.0 \\
\hline \multirow[t]{2}{*}{ TKN (\%TS) } & Ingestates & $3.45 \pm 0.61 \mathrm{a}$ & 2.75 & 5.15 \\
\hline & Digestates & $7.23 \pm 2.00 b$ & 4.43 & 11.00 \\
\hline \multirow[t]{2}{*}{ Ammonia (\%TS) } & Ingestates & $0.72 \pm 0.33 a$ & 0.06 & 1.39 \\
\hline & Digestates & $4.04 \pm 1.86 \mathrm{~b}$ & 1.74 & 8.19 \\
\hline \multirow[t]{2}{*}{$\mathrm{P}_{2} \mathrm{O}_{5}$ (\%TS) } & Ingestates & $0.77 \pm 0.35 a$ & 0.45 & 1.77 \\
\hline & Digestates & $2.39 \pm 0.44 b$ & 1.50 & 3.37 \\
\hline \multirow[t]{2}{*}{ TOC/TKN } & Ingestates & $13.1 \pm 1.8 b$ & 8.5 & 14.9 \\
\hline & Digestates & $5.6 \pm 1.7 \mathrm{a}$ & 3.1 & 9.7 \\
\hline \multirow[t]{2}{*}{ ADL (\%TS) } & Ingestates & $6.1 \pm 2.6 \mathrm{a}$ & 3.1 & 11.0 \\
\hline & Digestates & $20.5 \pm 5.7 b$ & 10.7 & 32.9 \\
\hline \multirow[t]{2}{*}{ Hemicell (\%TS) } & Ingestates & $3.4 \pm 1.3 \mathrm{a}$ & 0.9 & 5.4 \\
\hline & Digestates & $6.6 \pm 4.5 b$ & 0.0 & 13.9 \\
\hline \multirow[t]{2}{*}{ Cellulose (\%TS) } & Ingestates & $6.6 \pm 2.6 \mathrm{a}$ & 4.0 & 11.9 \\
\hline & Digestates & $9.1 \pm 3.4 \mathrm{~b}$ & 1.8 & 19.1 \\
\hline \multirow[t]{2}{*}{ CS (\%TS) } & Ingestates & $83.6 \pm 5.5 \mathrm{~b}$ & 70.7 & 89.7 \\
\hline & Digestates & $63.9 \pm 7.3 \mathrm{a}$ & 43.6 & 73.8 \\
\hline \multirow[t]{2}{*}{ ADF (\%TS) } & Ingestates & $12.7 \pm 4.9 \mathrm{a}$ & 7.1 & 21.4 \\
\hline & Digestates & $29.6 \pm 8.1 \mathrm{~b}$ & 13.4 & 45.1 \\
\hline \multirow[t]{2}{*}{$\mathrm{OD} 20\left(\mathrm{mg} \mathrm{O}_{2} \mathrm{~g}^{-1} \mathrm{TS}\right)$} & Ingestates & $219.1 \pm 60.3 \mathrm{~b}$ & 143.2 & 310.0 \\
\hline & Digestates & $90.8 \pm 28.1 \mathrm{a}$ & 13.6 & 143.2 \\
\hline \multirow[t]{2}{*}{$\mathrm{ABP}\left(\mathrm{Nml} \mathrm{g}^{-1} \mathrm{TS}\right)$} & Ingestates & $646.4 \pm 75.8 b$ & 498.0 & 772.0 \\
\hline & Digestates & $261.7 \pm 89.1 \mathrm{a}$ & 66.0 & 437.0 \\
\hline
\end{tabular}

${ }^{a}$ Means followed in the same column by the same letter are not statistically different $(p<0.05)$ according to Tukey's test.

\subsection{Specific oxygen uptake rate assay}

The specific oxygen uptake rate (SOUR) test is a biological aerobic assay. It is a measure of the oxygen uptake rate in a water solution during microbial respiration in degrading a suspended solid matrix. The microbial respiration works out in standardized moisture conditions and in maximized conditions of both oxygenation and bacteria-substrate interaction, amplifying the differences among different samples.

Dried and mechanically shredded samples $(\varnothing<1 \mathrm{~mm})$ underwent the SOUR test, which was performed following the method reported by Lasaridi and Stentiford (1998). Briefly, $0.2 \mathrm{~g}$ of dry matter was set in a flask to which the following were added: $500 \mathrm{ml}$ of deionized water, $12 \mathrm{ml}$ of phosphate buffer solution $\left(\mathrm{KH}_{2^{-}}\right.$ $\mathrm{PO}_{4} 0.062 \mathrm{~mol} \mathrm{l}^{-1}, \mathrm{~K}_{2} \mathrm{HPO}_{4} 0.125 \mathrm{~mol} \mathrm{l}^{-1}, \mathrm{Na}_{2} \mathrm{HPO}_{4} \cdot 7 \mathrm{H}_{2} \mathrm{O} 0.125$ $\mathrm{mol} \mathrm{l}^{-1} ; \mathrm{pH} \mathrm{7.2)}$, and $5 \mathrm{ml}$ of nutritive solution $\left(\mathrm{CaCl}_{2} 0.25 \mathrm{~mol} \mathrm{l}^{-1}\right.$, $\mathrm{FeCl}_{3} 0.9 \mathrm{mmol} \mathrm{l}^{-1}$ and $\mathrm{MgSO}_{4} 0.09 \mathrm{~mol} \mathrm{l}^{-1}$ ) made up according to the standard BOD test procedures (APHA, 1992). No nitrogen was added. During the test, standard conditions were maintained to ensure optimum microbial activity and reaction rates. To allow oxygen diffusion, the slurry was kept under agitation by using a magnetic stirrer and by performing intermittent aeration every 15 min. Potential oxygen uptake was reported as cumulative oxygen demand during the 20 -h test (OD20, $\mathrm{mg} \mathrm{O}_{2} \mathrm{~g} \mathrm{TS}^{-1} \mathrm{~h}^{-1}$ ).

\subsection{Statistical approach}

All statistical analyses were preceded by the determination of the normal distribution of the data set, as the successive linear regression and step wise regression analyses were based on the assumption of normality among distributions. Normal distributions were accomplished using the Shaphiro-Wilk test (ISO, 1994). Parameters that were not following normal distributions were normalized using the Box-Cox method (Box and Cox, 1964; Klemm et al., 2002). All statistical analyses were performed using the SPSS 13.0 package (SPSS International, Chicago, IL). Regression standard errors (SE) were reported as percentage of the mean $(M)$ of the observed data set $(\mathrm{SEM}=\mathrm{SE} / M \times 100)$ and were used as a qualitative indicator of the obtained linear regressions.

\section{Results and discussion}

\subsection{Chemical and biological parameters}

The results of all chemical analyses plus the OD20 respirometric test are reported in Tables 1 and 2. All parameters were observed to be influenced by the degradation degree of organic matter $(\mathrm{OM})$, which is directly related to retention time (RT) in the digesters of the biogas plant. All ingestates resulted in TS contents within the range of $11-20 \% \mathrm{w} / \mathrm{w}$, whereas those of digestates were found in the lower range $(3-12 \% \mathrm{w} / \mathrm{w})$. Characterization of total TS and biological parameters also showed differences in ingestates and digestates. ANOVA was performed to assess the significance of the RT as a factor influencing all parameters (Table 3 ).

As expected, RT was found to significantly $(P<0.001)$ influence most of the parameters, while for hemicellulose and cellulose, the significance was lower $(P<0.05)$. TS, VS, TOC, CS, OD20, ABP, and TOC/TKN showed lower means in the digestates, as a consequence of OM degradation. On the other hand, TKN, ammonia, ammonia/ TKN, ADF, ADL, cellulose, hemicellulose, and $\mathrm{P}_{2} \mathrm{O}_{5}$ were higher in the digestates, probably due to the concentration effect during digestion.

\subsection{Simple linear regressions of $A B P$ versus chemical and biological parameters}

Only some of the parameters, such as OD20, cellulose, TKN, and TOC/TKN, needed to be normalized for the statistical approach, while the others showed normal distributions.

Assuming that ABP was a dependent variable, significant $(P<0.001)$ positive regressions were found for VS, TOC/TKN, OD20, TOC, TS, and CS (Fig. 1). Negative significant regressions were shown by ammonia, TKN, ammonia/TKN, $\mathrm{P}_{2} \mathrm{O}_{5}, \mathrm{ADF}$, and ADL (Fig. 1). Very poor regression and lower significance levels $(P>0.05)$ were found for cellulose and hemicellulose contents (data not shown). The best regression was obtained for ABP vs. VS (model 1, Table 4), with a regression coefficient of 0.806 . The calculated SEM was around $23-24 \%$ (Table 4).

This result was unexpected as the literature suggests that ABP depends not only on VS content (quantitative aspect of the OM) but also on OM composition (e.g., content of carbohydrates, proteins, lipids, etc.) and OM quality (degradation efficiency; Chandler et al., 1979; Hashimoto, 1986; Robbins et al., 1979). In this work, as the considered set of samples included ingestates and the corresponding digestates, the main factor affecting ABP was the degree of OM degradation, which depends on the occurring processes (Table 3 ). Since the total OM content (approximately equal to VS content) was also affected by degradation, ABP showed good correlation with VS.

Volatile solid tests are very simple and less time-consuming analyses (within $24 \mathrm{~h}$ ) and they could be directly performed in the biogas plant itself. Plant operators could easily obtain a quick assessment of the ABP of both ingestate mixtures and digestate slurries. This could help them monitor the AD process by estimating degradation and biogas yields.

This method is particularly suitable as an "on-the-field method", but it does not consider parameters describing OM quality. On the other hand, correlations obtained (Fig. 1) suggested that, OD20, CS, $\mathrm{ADL}$, and $\mathrm{ADF}$, all describing OM quality, influence ABP. Habig (1985) reported regression models between $A B P$ and readily degradable fractions such as carbohydrate content $\left(R^{2}=0.83\right)$ and carbohydrate plus protein content $\left(R^{2}=0.92\right)$. Gunaseelan (2007) 

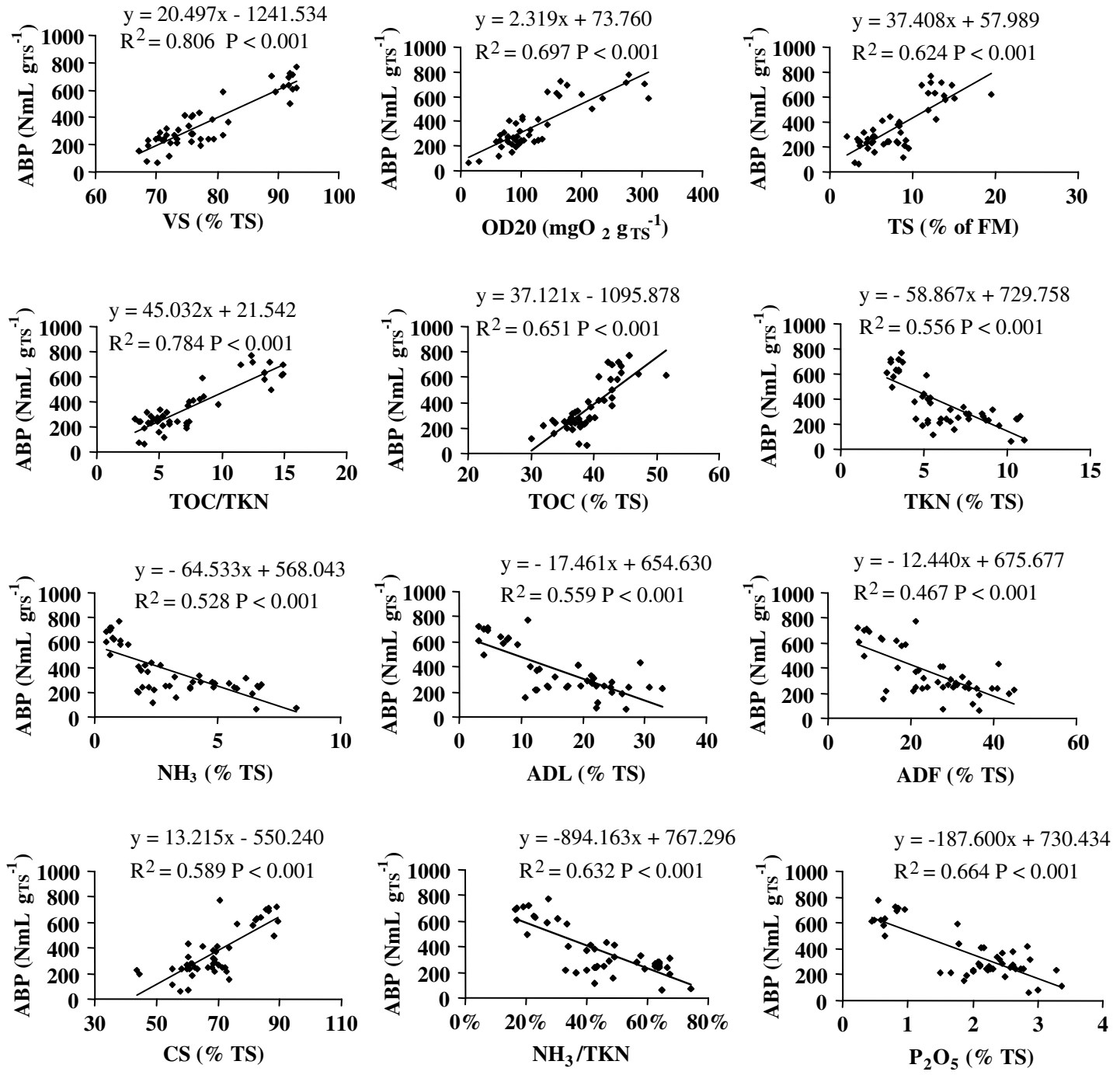

Fig. 1. Linear regressions between ABP and chemical and biochemical parameters, performed on the 46 samples studied.

Table 4

Multiple stepwise linear regression for predicting ABP

\begin{tabular}{|c|c|c|c|c|c|c|c|}
\hline Model & Equation & Variables involved & $R^{2}$ & Intercept & Slope & $P$ & SEM (\%) \\
\hline 1 & $\mathrm{ABP}=20.497 * \mathrm{VS}-1241.534$ & VS & 0.806 & -1241.534 & 20.497 & $<0.001$ & 23.5 \\
\hline 2 & $\mathrm{ABP}=13.782 * \mathrm{VS}+26.161 * \mathrm{OD} 20^{1 / 2}-997.890$ & $\begin{array}{l}\text { VS } \\
\text { OD20 }\end{array}$ & 0.880 & -997.890 & $\begin{array}{l}13.782 \\
26.161\end{array}$ & $<0.001$ & 18.7 \\
\hline 3 & $\mathrm{ABP}=10.480 * \mathrm{VS}+23.178 * \mathrm{OD} 20^{1 / 2}+10.979 * \mathrm{TOC}-1038.667$ & $\begin{array}{l}\text { VS } \\
\text { OD20 } \\
\text { TOC }\end{array}$ & 0.904 & -1038.667 & $\begin{array}{l}10.480 \\
23.178 \\
10.979\end{array}$ & $<0.001$ & 16.9 \\
\hline 4 & $\mathrm{ABP}=8.445 * \mathrm{VS}+19.173 * \mathrm{OD} 20^{1 / 2}+10.942 * \mathrm{TOC}+2.913 * \mathrm{CS}-1067.198$ & $\begin{array}{l}\text { VS } \\
\text { OD20 } \\
\text { TOC } \\
\text { CS }\end{array}$ & 0.918 & -1067.198 & $\begin{array}{l}8.445 \\
19.173 \\
10.942 \\
2.913\end{array}$ & $<0.001$ & 15.8 \\
\hline
\end{tabular}

confirmed this result by studying a series of vegetable waste and crops. The same author also showed how methane potential was correlated not only to VS and ash content but also to ADF content, cellulose, ADL, and TKN. Moreover, the literature confirms that the increase in ADL content increases the resistance of lignocellulosic material to anaerobic biodegradation (Chandler et al., 1979; Hashimoto and Chen, 1979; Robbins et al., 1979).

Therefore, more than just one parameter should be used to predict ABP. Gunaseelan (2007), for example, predicted ABP through multiple linear regression using carbohydrates, VS, ADF, ADL, and TKN $\left(R^{2}=0.90\right)$.

\subsection{Multiple stepwise linear regression}

To consider both quantitative and qualitative aspects of the $\mathrm{OM}$ and to obtain a more reliable model to predict ABP, a multiple stepwise linear regression was performed, maintaining $A B P$ as the dependent variable. Independent variables (all parameters studied) were introduced step by step, excluding nonsignificant ones. The results were three additional linear models, reported in Table 4. As expected, their $R^{2}$ coefficients increased at each step, while the SEM decreased.

Using VS and OD20 $0^{1 / 2}$ together as independent variables, a significant increase of the $R^{2}$ was obtained (0.880) and the SEM 
decreased to $18.7 \%$ (model 2). When TOC (normalized to natural logarithm; model 3) and CS (model 4) were also considered, relatively weak increases of the $R^{2}$ value were obtained (respectively, 0.904 and 0.918 ) and the SEM dropped to $16.9 \%$ and $15.8 \%$.

Model 2 may represent the best solution because it ensures a relatively high SEM decrease and it contains information about both total OM quantity (VS) and its degradability (OD20; D'Imporzano and Adani, 2007). At the same time, it requires reasonable laboratory work and only $24 \mathrm{~h}$ to conduct both analyses.

Models 3 and 4 are finer models predicting ABP as they consider with more depth both the quantitative (VS and TOC) and qualitative (OD20 and CS) characteristics of the OM. On the other hand, they would require much laboratory work without a satisfactory increase in estimation accuracy (SEM).

\section{Conclusions}

A statistical model for predicting ABP using quicker laboratory analysis was studied. An increasing number of the four most significant variables (VS, OD20, TOC, and CS) were used, representing both OM content and OM quality of the matrices. Four linear models were proposed, with $R^{2}$ coefficients ranging between 0.844 and 0.913 and the SEM between $16 \%$ and $23 \%$.

To predict biogas potentials and assess degradation yields of the observed biogas plant, the linear regressions obtained can be used, depending on the needed accuracy and the number of laboratory analyses to be performed on the ingestates and digestates. As the number of analyses would be reduced, the most convenient solution could be achieved using only OD20 and VS (model 2); moreover, with an SEM around 19\%, results could be relatively satisfactory in evaluating AD process performance, within operational time schedules.

\section{References}

Adani, F., Calcaterra, E., Malagutti, L., 2001. Preparation of a test for estimating biogas production from pretreated urban waste. In: Christensen, T.H., Cossu, R., Stegman, R. (Eds.), The Sustainable Landfill, Eighth International Waste Management and Landfill Symposium, S. Margherita di Pula, Cagliari, Italy. CISA, Cagliari, Italy, pp. 571-577.

APHA - American Public Health Association, 1992. Standard Methods for the Examination of Water and Wastewater, 18th ed. APHA, Washington, DC.

APHA - American Public Health Association, 1998. Standard Methods for the Examination of Water and Wastewater, 20th ed. APHA, Washington, DC.

Bjorndal, K.A., Moore, J.E., 1985. Prediction of fermentability of biomass feedstocks from chemical characteristics. In: Smith, W.H. (Ed.), Biomass Energy Development. Plenum Press, New York, pp. 447-454.

Box, G.E.P., Cox, D.R., 1964. An analysis of transformation. J. Royal Stat. Soc. 26 (B), 211-243.
Chandler, J.A., Jewell, W.J., Gossett, J.M., Van Soest, P.J., Robertson, J.B., 1979. Predicting methane fermentation biodegradability. Biotechnol. Bioeng. Symp. 10, 93-107.

Chynoweth, D.P., Owens, J.M., Legrand, R., 2001. Renewable methane from anaerobic digestion of biomass. Renew. Energ. 22 (3), 1-8.

D'Imporzano, G., Adani, F., 2007. The contribution of water soluble and water insoluble organic fractions to oxygen uptake rate during high rate composting. Biodegradation 18, 103-113.

Gunaseelan, V.N., 1997. Anaerobic digestion of biomass for methane production: a review. Biomass Bioenerg. (1/2), 83-114.

Gunaseelan, V.N., 2007. Regression models of ultimate methane yields of fruits and vegetable solid wastes, sorghum and napiergrass on chemical composition. Bioresour. Technol. 98, 1270-1277.

Habig, C., 1985. Influences of substrate composition on biogas yields of methanogenic digesters. Biomass 8, 245-253.

Han, Y.W., Lee, J.S., Anderson, A.W., 1975. Chemical composition and digestibility of ryegrass straw. J. Agric. Food Chem. 23, 928-931.

Hansen, T.L., Schmidt, J.E., Angelidaki, I., Marca, E., Jansen, J.I.C., Mosbæk, H., Christensen, T.H., 2004. Method for determination of methane potentials of solid organic waste. Waste Manage. 24, 393-400.

Hashimoto, A.G., 1986. Pretreatment of wheat straw for fermentation to methane. Biotechnol. Bioeng. 28, 1857-1866.

Hashimoto, A.G., Chen, Y.R., 1979. Anaerobic fermentation of beef cattle and crop residues, in Golden, CO. In: Proceedings of the Third Annual Fuels from Biomass Symposium, June, pp. 4-6.

Holm-Nielsen, J.B., Dahl, C.K., Esbensen, K.H., 2006. Representative sampling for process analytical characterization of heterogeneous bioslurry systems - a reference study of sampling issues in PAT. Chemometr. Intell. Lab. Systems 83, $114-126$.

IRSA CNR, 1994. Metodi analitici per le acque, Quaderni, N. 100. Istituto Poligrafico e Zecca dello Stato, Rome, Italy.

ISO, 1994. Accuracy (Trueness and Precision) of Measurement Methods and Results. ISO 5725-5732.

Klemm, D.J., Blocksom, K.A., Thoney, W.T., Fulk, F.A., Herlihy, A.T., Kaufmann, P.R., Cormier, S.M., 2002. Method development and use of macroinvertebrates as indicators of ecological conditions for streams in the mid-Atlantic highlands region. Environ. Monit. Assess. 78, 169-212.

Lasaridi, K.E., Stentiford, E.I., 1998. A simple respirometric technique for assessing compost stability. Water Res. 32 (12), 3717-3723.

Muller, W., Fricke, K., Vogtmann, H., 1998. Biodegradation of organic matter during mechanical biological treatment of MSW. Compost Sci. Util. 6 (3), 42-52.

Nousiainen, J., Ahvenjarvi, S., Rinne, M., Hellamaki, M., Huhtanen, P., 2004. Prediction of indigestible cell wall fraction of grass silage by near infrared reflectance spectroscopy. Animal Feed Sci. Technol. 115, 295-311.

Robbins, J.E., Armold, M.T., Lacher, S.L., 1979. Methane production from cattle waste and delignified straw. Appl. Environ. Microbiol. 38, 175-177.

Sanderson, M., Agblevos, F., Collins, M., Johnson, D.K., 1996. Compositional analysis of biomass feedstocks by near-infrared reflectance spectroscopy. Biomass Bioenerg. 11 (5), 365-437.

Tong, X., Smith, L.H., Mc Carty, P.L., 1990. Methane fermentation of selected lignocellulosic materials. Biomass 21, 239-255.

Van Soest, P.J., Robertson, J.B., Lewis, B.A., 1991. Methods for dietary fibers, neutral detergent fiber and non-starch polysaccharides in relation to animal nutrition. J. Dairy Sci. 74, 3583-3597.

Wilman, D., Fiels, M., Lister, S.J., Givens, D.I., 2000. The use of near-infrared spectroscopy to investigate the composition of silages and the rate and extent of cell-wall degradation. Animal Feed Sci. Technol. 88, 139-151.

Zhang, R., El-Mashad, H.M., Hartman, K., Wang, F., Liu, G., Choate, C., Gamble, P., 2007. Characterization of food waste as feedstock for anaerobic digestion. Bioresour. Technol. 98, 929-935. 